\title{
On the Mode of Administering a Questionnaire and Responses to Open-Ended Items
}

\author{
ROBERT M. GRoves
}

Survey Research Center, University of Michigan

\begin{abstract}
Concurrently administered personal and telephone surveys are compared to measure differences between the modes for identical questions. Speed of questioning is found to be greater in telephone interviews than personal interviews. The faster pace of telephone interviews is linked to shorter answers to open-ended items on the telephone. The tendency to reduce such responses is disproportionately exhibited by younger, affluent respondents who tend to provide detailed responses in personal interviews.
\end{abstract}

In a recent experimental study of the relative efficiency of telephone and personal interview surveys, it was casually observed that the pace of the telephone interviews seemed faster than that of typical personal interviews. Although it was not always clear whether the respondent or the interviewer set the pace, it was hypothesized that the different delivery styles might be associated with method effects between personal and telephone interviews. Since the same set of questions was asked of adult respondents on the telephone and in person, the project offered an opportunity to investigate: (1) whether the average speed of telephone interviews tended to be greater than personal interviews asking the same questions; that is, whether greater speed could be viewed as a characteristic of phone interviews; (2) whether speed of the interview is associated with method differences in the number of responses given to open-ended items; (3) whether the number of responses to open-ended items varies predictably according to the method of administration; (4) whether that variation is related to differences in the categories of responses given; and (5) whether there are subgroups of the adult population that disproportionately reflect method effects in their number of responses to openended items. Although there have been other studies which examined

Work supported by NSF (Soc 76-07519). The author's appreciation to Stanley Presser and Charles Cannell for comments on an earlier draft of this paper. Address for reprint requests: Robert M. Groves, Survey Research Center, University of Michigan, Ann Arbor, Michigan 48106. 
differences in results between telephone and personal interviews (e.g., Hochstim, 1967; Colombotos, 1965; Rogers, 1976; and others), the recent SRC study on a national sample of adult respondents provides an updating of the measurement properties of the two modes of administration. This paper examines one set of measurement-related characteristics of personal and telephone interviews.

The ultimate dependent variable in the analysis is the difference between the answers to open-ended items received in telephone and personal interviews. This measure is seen as a proxy for a bias measure. Since there is no externally available population value for the statistics we examine, measurement of bias is impossible. Instead we analyze the difference between the modes of administration and attempt to explain variation in the magnitude of the difference. This difference is viewed as a function of the number of responses to open-ended questions. That is, in some sense, the magnitude of response is related to the type of response given. The pace of the interview is viewed as both a cause and a result of the number of answers; and both the speed of the interview and the magnitude of response are seen as functions of certain demographic characteristcs of the respondent and of his attitudes toward the particular interview. We will first observe method differences in the speed of the interview and in the number of responses to open-ended items. After showing that the number of responses given is related to differences in the distribution of answers, we will construct multivariate models that attempt to explain variation between modes of administration in the number of answers given to open-ended questions.

\section{THE RESEARCH DESIGN}

The data analyzed in this paper come from a methodological study conducted in April and May, 1976, at the Survey Research Center. The study was designed to compare the sampling, field, and measurement properties of telephone and personal interview surveys. Personal interviews with adult respondents (18 years or older) in 1548 sample households were collected by 132 interviewers in the 74 primary areas of the SRC national sample. At the same time, 38 interviewers at the Ann Arbor SRC office collected telephone interviews from adult respondents in 1734 households, with telephones sampled by random-digit dialing. ${ }^{1}$ Identical definitions of an eligible respondent and identical objective selection procedures for respondents were applied in both samples. The response rate for the personal interview survey was $74.2 \%$; for the telephone survey the response rate ranged from 59 to $70 \%$, depending on whether or not unanswered sample numbers are included as working household

\footnotetext{
' Details of the sample designs can be found in Groves and Kahn (forthcoming).
} 
numbers. ${ }^{2}$ Identical questions were asked of the personal and telephone samples, and the interviewing work for the two samples was concurrent. An additional set of questions was asked of personal interview respondents, but it was placed after all the questions that were identical in both modes. The populations of the two samples differ only in that the personal interview sample included some respondents in households without telephones. Combining results for the telephone and personal interview samples for respondents in households with telephones creates a sample of that population with measures differing only in their method of collection. Thus we can measure method effects unhampered by different population representation, but our inference is limited to the adult population in households with telephones.

\section{DATA ANALYSIS}

The second section of the questionnaire in both telephone and personal interviews contained attitudinal questions about life satisfaction, political and social issues, and factual items concerning voting behavior and income tax filing experiences. Two sets of questions in the personal interviews involved "show cards" which listed the possible responses to the questions. These cards were replaced in the telephone interview by more lengthy introductions describing the response scales. This adaptation probably increased the time required for this section in the telephone interviews. Nonetheless, the median time for this section of questions was 11.8 minutes on the telephone and 13.7 minutes in personal interviews. The same questions thus took about $16 \%$ longer to complete in person. ${ }^{3}$

One explanation of the speed of telephone questioning is the elimination of extraneous conversation which sometimes occurs in face-to-face interviews. The interaction on the telephone involves less intrusion into the respondent's home; for example, the interviewer doesn't have to enter the respondent's living room as a guest, and the social amenities that would accompany such an event are dispensed with. The interview on the telephone is also relatively private, and only respondent and interviewer hear both question and answer. Thus, other household members have fewer reasons to comment on questions or answers. Furthermore, only audio cues are relevant; there are no visual distractions for the interviewer, so she can concentrate on the questionnaire itself. This view suggests that the nonproductive "chit chat" which occurs in personal

\footnotetext{
${ }^{2}$ Work on a later project has shown that 19 out of 20 of the sample numbers not answered after 17 calls were nonworking numbers. This figure is applicable only to national stratified element RDD samples and is based on a very small sample size. The result should be treated cautiously.

${ }^{3}$ These time estimates are sometimes subject to rather large interviewer recording errors. The specific estimates above were made only after many cases were deleted because of recording errors.
} 
interviews is reduced in telephone interviews. We have no data for a direct test of this hypothesis, but we do note a rival notion: The speed of telephone interviews relative to personal interviews reflects not a more efficient use of interview time, but a reduction of time available to respondents to think about and to fully reveal feelings about the questions. In short, the speed of telephone interviews may yield more superficial responses to questions.

The study does provide some data on the effects of the faster pace of telephone interviews, and these data arise from open-ended items contained in the questionnaire. Open-ended measures allow the respondent to articulate his own answer to a question. The number of different responses that a person gives to open-ended questions in the two modes of data collection may be a useful proxy variable for the amount of effort respondents invest in the task of answering, or the extent to which the answer fully characterizes the respondent's thoughts. Only the first two mentioned answers are routinely coded for open-ended items, and Table 1 presents the percentage of respondents, among those answering the ques-

TABLE

Percentage of Respondents Answering Open-Ended Questions Who Gave Morc than One Mention by Mode of Interview"

\begin{tabular}{|c|c|c|c|}
\hline Subject of open-ended item & $\begin{array}{l}\text { Telephone } \\
\text { interviews }\end{array}$ & $\begin{array}{l}\text { Personal } \\
\text { interviews } \\
\text { (phone } \\
\text { households) }\end{array}$ & $\begin{array}{c}\text { Percentage } \\
\text { difference } \\
\text { (personal-phone } \\
\text { interviews) }\end{array}$ \\
\hline $\begin{array}{l}\text { 1. Reasons for feelings about } \\
\text { family's future economic } \\
\text { status }\end{array}$ & $\begin{array}{c}27.6 \\
(1316)\end{array}$ & $\begin{array}{c}34.1 \\
(1132)\end{array}$ & $6.5^{*}$ \\
\hline $\begin{array}{l}\text { 2. Type of economic news } \\
\text { recently heard }\end{array}$ & $\begin{array}{l}34.1 \\
(906)\end{array}$ & $\begin{array}{l}34.2 \\
(728)\end{array}$ & 0.1 \\
\hline $\begin{array}{l}\text { 3. Reasons for opinions about } \\
\text { current conditions for } \\
\text { buying a house }\end{array}$ & $\begin{array}{l}27.1 \\
(1463)\end{array}$ & $\begin{array}{c}35.1 \\
(1252)\end{array}$ & $8.0^{*}$ \\
\hline $\begin{array}{l}\text { 4. Reasons for opinions about } \\
\text { conditions for buying a } \\
\text { car in the next } 12 \text { months }\end{array}$ & $\begin{array}{c}22.9 \\
(1184)\end{array}$ & $\begin{array}{c}25.7 \\
(1071)\end{array}$ & 2.8 \\
\hline $\begin{array}{l}\text { 5. Reasons for opinions about } \\
\text { current conditions for } \\
\text { buying major household } \\
\text { items }\end{array}$ & $\begin{array}{c}11.3 \\
(1230)\end{array}$ & $\begin{array}{c}14.2 \\
(1073)\end{array}$ & 2.9 \\
\hline $\begin{array}{l}\text { 6. Type of most important } \\
\text { problems facing the } \\
\text { country }\end{array}$ & $\begin{array}{c}56.3 \\
(1537)\end{array}$ & $\begin{array}{c}65.5 \\
(1366)\end{array}$ & $9.2^{*}$ \\
\hline
\end{tabular}

" Total numbers of respondents giving at least one mention appear in parentheses below percentages. There are totals of 1734 respondents in the telephone sample and 1440 in phone households with personal interviews. Those respondents absent from the figures above had missing data or answered "Don't Know" on the open-ended questions.

"First of a multi-probe question series.

$* p<.05$ based on standard errors adjusted for average design effects of the two samples. 
tion, who gave at least two answers (i.e., "Don't knows," "INAPs," and "not ascertains" are excluded). Results for six different open-ended questions consistently show that a smaller proportion of respondents give multiple mentions on the telephone than in personal interviews. The difference is negligible $(0.1 \%)$ for some questions-for example, Question 2 about the nature of economic news recently heard-but is rather large (9.2\%) for other questions-for example, Question 6 about the most important problems facing the country.

The "most important problems" question was included in the survey as a direct test of the hypothesis that respondents would tend to answer open-ended items more superficially on the telephone than in person. Both telephone and personal interviewers were instructed to use only the probing specifically written into the questionnaire. The question appeared as:

B6. What do you think are the most important problems facing this country?

B6a. What other important problems are there?

B6b. (IF PROBLEMS MENTIONED IN B6a) Are there any other important problems?

Two mentions were coded for each of the three sections of the question, unless all answers to that section fell into the same one of 13 response categories. In addition, coders counted the number of different problems mentioned and whether or not they fell into the same category. Figure 1 plots separately the distribution of number of answers to the question for telephone and personal interviews. It demonstrates that the proportion of respondents giving more than two total answers on all parts of this question is larger in personal interviews $(69.6 \%)$ than in telephone interviews (58\%). This is consistent with the results exhibited in Table 1 on five other open-ended questions but is more detailed since it compares complete counts of answers to an open-ended item.

We have shown that the telephone and personal interview samples differ in speed of administration and in number of mentions to open-ended items. We now observe that the number of items mentioned is related to the distribution of the substantive responses to the question. Table 2 presents separately percentage distributions of problems mentioned in 


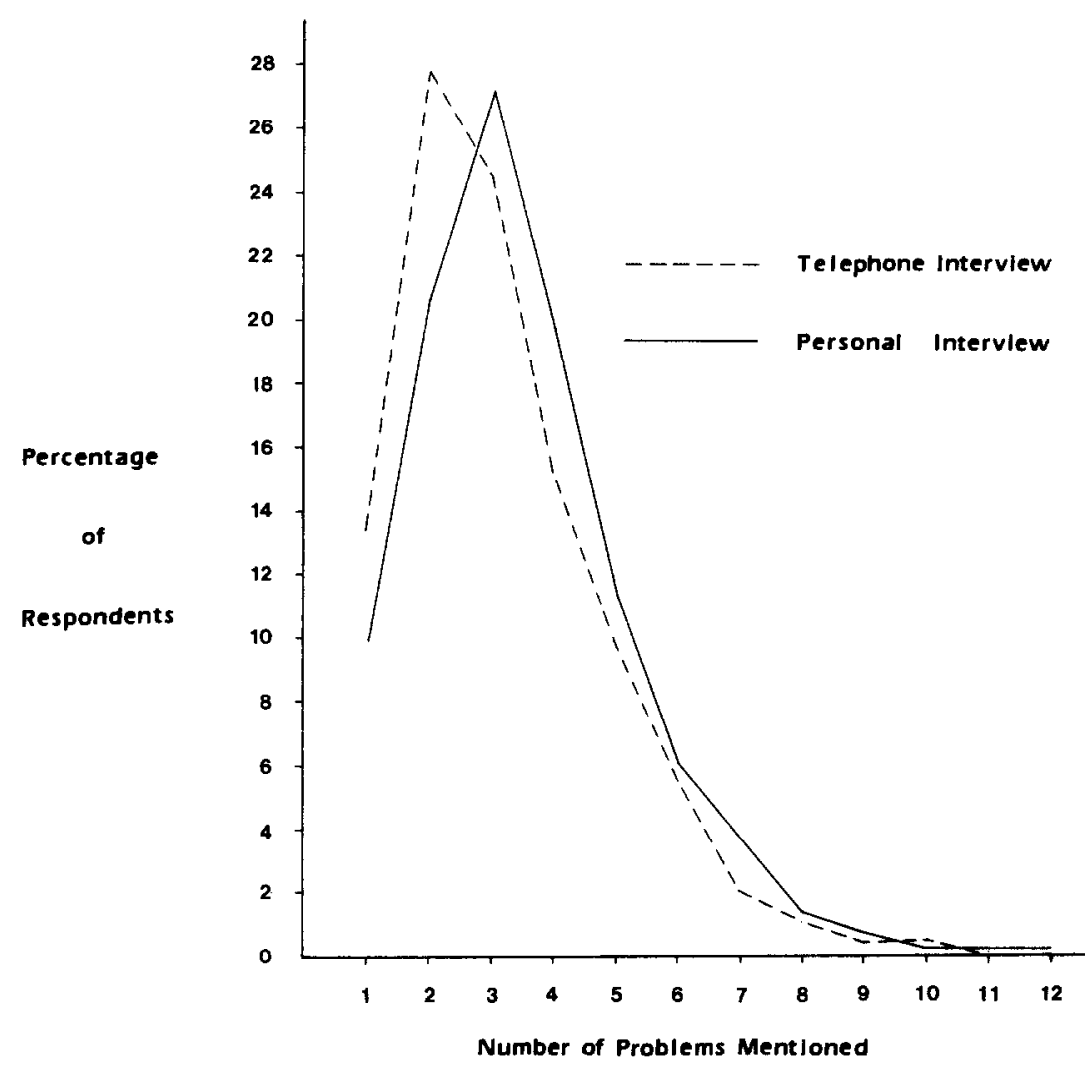

FIG. 1. Percentage distribution of number of problems mentioned on "Most Important Problems" question by mode of interview.

phone and personal interviews, among those mentioning less than three and among those giving three or more problems. The unit of analysis in this table is not the respondent but rather the response; a respondent who mentions three different problems is counted three times in the table. At the bottom of the table the coefficients of dissimilarity compare the distributions of the telephone and personal interview samples. ${ }^{4}$ They show that there are larger method effects $(\Delta=.093)$ among those giving few answers than among those giving many answers $(\Delta=.080)$. These effects are smaller than anticipated but in the hypothesized direction. Respondents giving few problems tend to omit mentions of social welfare problems much more frequently than do respondents who give many answers.

${ }^{4}$ Note that the coding instructions for these items specified coding of second mentions for the three parts only if the second mention fell into a different category of problem than that of the first mention. There was no such restriction across the different parts of the question. The result is a coding of threc pairs of problems, each pair containing two different types of problems. This coding design forces second mentions in each section into different categories and smooths the distribution of answers somewhat. The fact that the three sections together are not restricted in repeating codes makes the above comparison useful. 


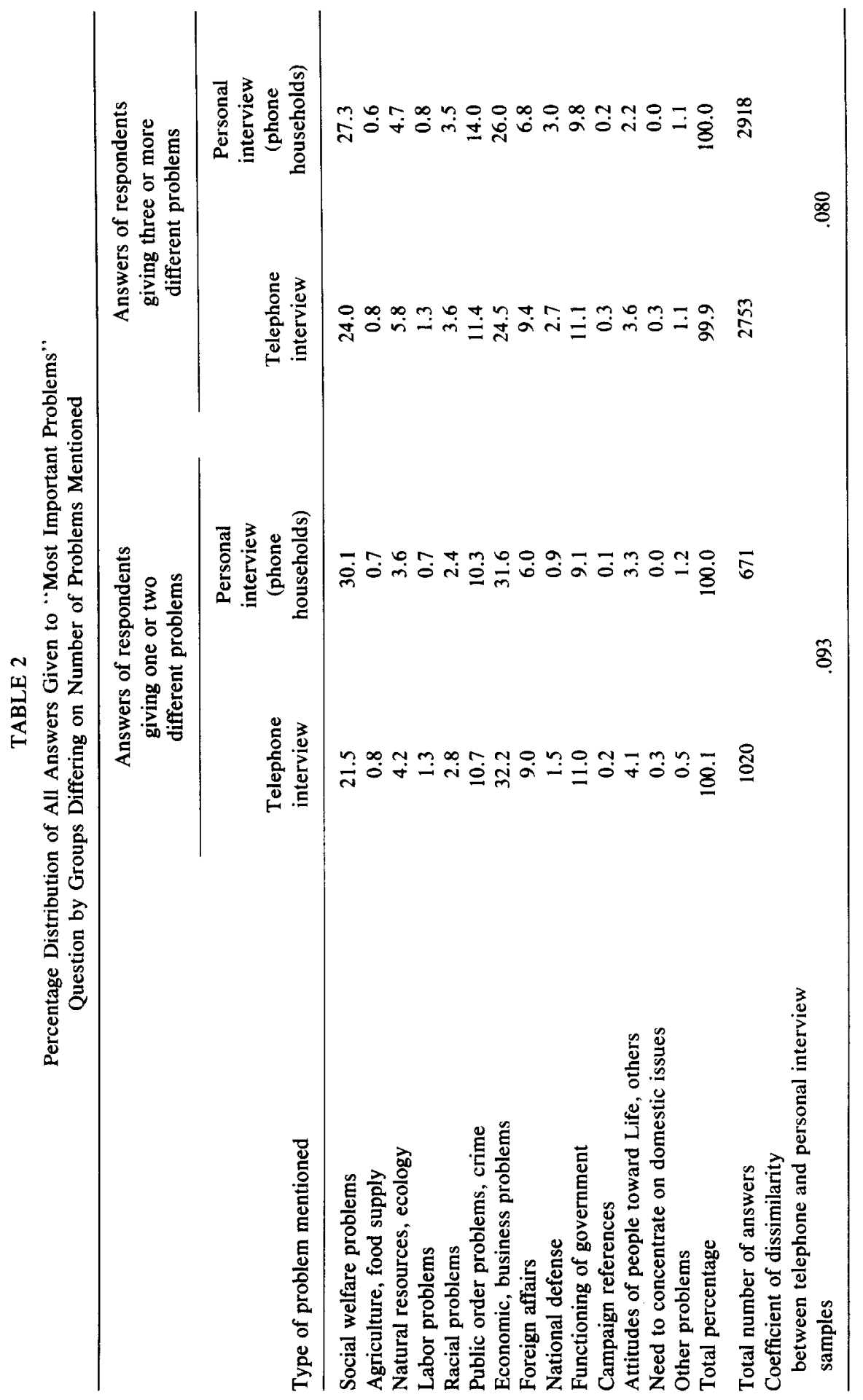


TABLE 3

Percentage of Respondents Mentioning Three or More Problems by Length of Questioning in Minutes and Mode of Administration ${ }^{a}$

\begin{tabular}{lccc}
\hline & \multicolumn{2}{c}{$\begin{array}{c}\text { Percentage of respondents } \\
\text { mentioning three or more problems }\end{array}$} & \\
\cline { 2 - 3 } Length of section & $\begin{array}{c}\text { Phone } \\
\text { interviews }\end{array}$ & $\begin{array}{c}\text { Personal } \\
\text { interviews } \\
\text { (phone household) }\end{array}$ & $\begin{array}{c}\text { Difference } \\
\text { personal-phone } \\
\text { interviews }\end{array}$ \\
\hline 10 minutes or less & 47.6 & 59.7 & 12.1 \\
$11-15$ minutes & $(460)$ & $(288)$ & 11.0 \\
$16-20$ minutes & 59.5 & 70.5 & 8.0 \\
21 or more minutes & $(629)$ & $(567)$ & \\
& 67.4 & 75.4 & -3.1 \\
\hline
\end{tabular}

a 'Total numbers of respondents mentioning three or more problems appear in parentheses below percentages.

This difference is greater among telephone respondents than among personal interview respondents. Data analysts often utilize only the first response to open-ended items on the assumption that the first mentioned is the most important to the respondent. The inference is the same, however, if we restrict our comparison to the first mentioned problem: There are larger method effects on first response for those that mention few problems $\left(\Delta=.150, \tau=.0071, p_{\chi^{2}}=.0039\right)$ than for those giving many problems $\left(\Delta=.111, \tau=.0031, p_{X^{2}}=.0016\right) .{ }^{5}$ Finally, we note that the speed of the interview affects the number of problems mentioned in response to the open-ended question. Table 3 presents the percentage of respondents mentioning three or more problems by mode of administration within groups requiring the same times for that section of questions. This table shows that among those requiring less than 10 minutes for the questions, $12.1 \%$ fewer people gave more than three answers in telephone interviews than in personal interviews; the difference is reduced in groups requiring longer times. Indeed, among those requiring 21 minutes or more to complete the section, proportionately more telephone than personal respondents mentioned many problems. The slower the pace of questioning, the smaller the method effects.

We see, therefore, that the telephone interviews were generally conducted at a more rapid pace, that more rapid interviews tended to elicit fewer mentions, and even worse, that this tendency was exaggerated on the telephone. The greatest method effects are found in the group with the

s These probability values are based on simple random sample estimates and should be adjusted for departures of the two sample designs from simple random samples. Average inflation factors for the standard errors of means for the personal sample are about 1.15. 
shortest interviews. Similarly, the number of problems mentioned tends to be fewer on the phone, and the largest method effects on type of problem mentioned are found among those mentioning few problems. The time required to complete the questions is partially a function of the richness of response to the open-ended item we are examining, and attempting to control the pace of the interview on the telephone may be more difficult than directly attacking the problem of respondents giving fewer responses to open-ended items. If we can identify types of respondents who exhibit the largest method effects in responses to open-ended items, we may provide guidance in choosing a mode of administering a questionnaire to a particular population and also suggest areas where further research on telephone interviewing techniques would yield the greatest success.

\section{RESPONDENT GROUPS EXHIBITING DIFFERENT METHOD EFFECTS ON NUMBER OF PROBLEMS MENTIONED}

In this section we will attempt to identify subgroups of the population for which the telephone interview yielded much lower frequencies of problems mentioned. Two types of variables, demographic and attitudinal, will be utilized. Since demographic variables are often used as definitions of the population of inference, this analysis could help determine appropriate modes of administration for interviews when studying different subpopulations. In the set of demographic variables we include age, sex, education, and income. Several measures in the data reflect respondent attitudes about the interview itself; we utilize self-reported uneasiness about answering different kinds of questions as well as interviewer judgments of the respondent's degree of suspicion toward the interview and of the respondent's interest in the interview. ${ }^{6}$ Finally, we include the interviewer's judgment of the respondent's ability to articulate his thoughts. This second set of variables, labeled "respondent reactions," is included to investigate whether among groups sharing the same demographic characteristics there may be respondent reactions that are related to the telephone respondents' tendency toward fewer mentions. If these reactions can be observed by the interviewer, then the problem of fewer responses on the phone may yield itself to experimental study of interviewing styles that could reduce its magnitude.

The dependent variable in the analysis is a dichotomy separating those who mentioned one or two problems in total for all sections of the "most important problems" questions from those that mentioned three or more

${ }^{6}$ The last series of questions in both the telephone and personal interviews asked the respondent if he felt uneasy about answering questions concenring: (1) his income, (2) his racial attitudes, (3) his income tax returns, (4) his health, (5) his job, (6) his voting behavior, and (7) his political opinions. A scale was formed by counting the number of question types about which the respondent felt uneasy. The interviewer judgments were made in an "interviewer observation" section after the interview was completed. 
problems. The overall table showing the realtionship between mode of administration and the number of responses is Table 4 . This is merely a collapsing of the results presented in Fig. 1 and shows that a larger proportion of personal interview respondents $(69.6 \%)$ mention three or more problems than do respondents in telephone interviews (58.7\%). The multivariate analysis that follows examines whether the relationship between mode of administration and number of mentions is different for various demographic and attitudinal subgroups.

Respondents's education (coded less than high school, high school, more than high school), respondent's age (coded 18-30,31-50, 51 or more), and total family income (coded less than $\$ 7,500, \$ 7,500-\$ 15,000$, more than $\$ 15,000$ per year) each singly appear to affect the relationship between mode and numbers of mentions. Table 5 shows surprising results: It is the "best" respondents that exhibit the largest method effects. For example, the difference in proportions giving many answers is $10.6 \%$ among those in the lowest educational group, but $12.3 \%$ for those in the highest educational group. However, in both modes the highest educational group generally gives larger numbers of responses than the lowest educational group. Similarly the group with the highest total family income tends to mention more problems in either mode relative to those with lower incomes, but also exhibits larger differences in that behavior by mode of interview (13.8\%) than does the lowest income group (7.1\%). There were no apparent sex differences in method effects; both men and women tend to mention fewer problems in the telephone interview. Respondents over 50 years of age show smaller differences $(6.1 \%)$ due to mode of interview than younger respondents ( $14.0 \%$ or more). Two "respondent reaction" variables had no effect on the relationship between mode of administration and number of problems mentioned: These were the respondent's self-perceived uneasiness about answering certain questions and the interviewer's perception of whether the respondent appeared suspicious about the interview. Those judged most articulatc, however, exhibited smaller method effects on number of responses $(10.5 \%)$ than did those judged to have a "limited vocabulary" (17.1\%). Conversely those judged to have "very high interest" in the interview exhibited larger effects $(14.4 \%)$ than those judged to have little interest (7.9\%). The results tend to show that the people generally thought to perform best as respondents exhibit the greatest behavior differences in the two modes. ${ }^{7}$

\footnotetext{
' We should note the possibility of "ffoor" effects producing these results. If we view the telephone interview as a suppressor of natural tendencies to mention problems in response to the question, then for those that tend to mention few problems naturally, the effects of the phone are limited by mentioning at least one problem. This does not appear to be a large problem in these data. The smallest subgroup percentage giving three or more problems in person is $54.8 \%$; there is large opportunity to reduce that before the limiting point of $0 \%$ is reached.
} 
TABLE 4

Number of Problems Mentioned in "Most Important Problems" Question by Mode of Administration

\begin{tabular}{lcccccc}
\hline & \multicolumn{5}{c}{ Number of problems mentioned } \\
\cline { 2 - 5 } Mode of administration & $\begin{array}{c}\text { One or } \\
\text { two }\end{array}$ & $\begin{array}{c}\text { Three } \\
\text { or more }\end{array}$ & Total & $N$ & $\begin{array}{c}\text { Missing } \\
\text { data }\end{array}$ \\
\hline $\begin{array}{l}\text { Telephone } \\
\text { Personal }\end{array}$ & $41.3 \%$ & $58.7 \%$ & $100.0 \%$ & 1534 & 200 \\
& $30.4 \%$ & $69.6 \%$ & $100.0 \%$ & 1367 & 72 \\
\hline
\end{tabular}

We first use the demographic variables to construct multivariate models on the contingency tables measuring method effects on number of responses. Then we add the "respondent reaction" variable to the important demographic predictors. The demographic variables found to be related singly to method effects were included in a multivariate contingency table analysis, and using the ECTA program, log linear models were constructed to determine the unique effects of these demographic

TABLE 5

Percentage of Those Answering Who Mentioned Three or Morc Problems by Five Independent Variables by Sample Type

\begin{tabular}{|c|c|c|c|c|}
\hline \multirow{2}{*}{\multicolumn{2}{|c|}{ Subgroup }} & \multicolumn{2}{|c|}{ Sample type } & \multirow{2}{*}{$\begin{array}{c}\text { Difference } \\
\text { (personal-phone) }\end{array}$} \\
\hline & & Phone & Personal & \\
\hline \multirow[t]{4}{*}{1.} & Respondent's education & & & \\
\hline & $0-11$ years & 44.2 & 54.8 & 10.6 \\
\hline & High school diploma & 55.5 & 68.6 & 12.1 \\
\hline & 13 or more years & 69.6 & 81.9 & 12.3 \\
\hline \multirow[t]{4}{*}{2.} & Total family income & & & \\
\hline & $0-7500$ dollars & 52.1 & 59.2 & 7.1 \\
\hline & $7500-15,000$ dollars & 59.1 & 71.3 & 12.2 \\
\hline & 15,000 dollars or more & 64.0 & 77.8 & 13.8 \\
\hline \multirow[t]{4}{*}{3.} & Respondent's age & & & \\
\hline & $18-30$ years & 58.0 & 72.1 & 14.1 \\
\hline & $31-50$ years & 62.6 & 76.6 & 14.0 \\
\hline & 50 or more years & 56.4 & 62.5 & 6.1 \\
\hline \multirow[t]{6}{*}{4.} & $\begin{array}{l}\text { Ability to articulate } \\
\text { thoughts }\end{array}$ & & & \\
\hline & One on scale-very & & & \\
\hline & articulate & 72.8 & 83.3 & 10.5 \\
\hline & Two on scale & 58.0 & 69.4 & 11.4 \\
\hline & Three or four- & & & \\
\hline & limited vocabulary & 39.1 & 56.2 & 17.1 \\
\hline \multirow[t]{4}{*}{5.} & Interest in interview & & & \\
\hline & Very high & 71.8 & 86.2 & 14.4 \\
\hline & Above average & 64.7 & 76.4 & 11.7 \\
\hline & Average to very Low & 52.2 & 60.1 & 7.9 \\
\hline
\end{tabular}


correlates. ${ }^{8}$ Table 6 presents the results of model-fitting on the five-way table containing number of problems mentioned $(\mathrm{N})$, mode of administration (M), total family income (I), respondent's age (A), and respondent's education $(E)$. The categories for the five variables are those mentioned above, so the table contains $2 \times 2 \times 3 \times 3 \times 3=108$ cells.

In fitting models to this five-way table, we are most interested in terms which specify that the relationship between mode of administration and number of responses is different for different categories of the demographic variables. These terms are the three-way interaction terms denoted by NMI, NMA, and NME. The first model ( 1 on Table 6) includes all three of these interaction terms, the term measuring interactions among mode of administration and all demographic variables (MIAE), and all direct and interaction effects of the demographic variables on the number of responses (NIAE). This model fits the data rather poorly ( $p=$ .379), and later models show that some of the terms included in model 1 are extraneous to the fit. Through a series of successive models we learn that education groups show no significant differences in their method effects (i.e., NME is not significant to the fit of the model; compare models 2 and 1). Categories of age and family income seem to exhibit different method effects, but models containing both interaction terms do not fit the data significantly better than models with each singly. The base model to which we compare models containing method effects for income and age groups is MIAE, NIAE, NM, (model 8) which specifies that income, education, and age affects the number of problems mentioned in the same way for both modes, and the method effects that do exist are the same for all subgroups. This model fits the data reasonably well, $\chi^{2}=$ $25.01, d f=26, p>$.5. Indeed, comparing models 5-7 to model 8 shows that none of the terms. NMA, NMI, or NME, contributes significantly to the fit of the model. For example, once the direct effects of income and education on number of mentions are controlled, age groups do not exhibit significantly different method effects. Similar statements can be made about the other variables controlling on the remaining two. The percentage differences observed in Table 5 disappear when other demographic variables are controlled. The most attractive model is probably model 13, MIAE, NM, NI, NA, and NE, incorporating the same method effect for all demographic subgroups, NM. Although this model suggests that the subgroup differences in Table 5 disappear when proper controls are introduced, the researcher cannot introduce these controls in most work and must deal with the gross effects of the variables.

Although none of the demographic variables usefully predicted method effects, it is helpful to include a representative of the demographic mea-

* For a description of this analysis technique see L. A. Goodman (1971), "The multivariate analysis of qualitative data: Interactions among multiple classifications." Journal of the American Statistical Association 65, 226-56. 
TABLE 6

Log Linear Models Fit to the Five-Way Contingency Table Including Number of Responses $(\mathrm{N})$, Mode of Administration (M), Family Income (I),

Respondent's Age (A), and

Respondent's Education (E) ${ }^{a}$

\begin{tabular}{llrcc}
\hline Model & \multicolumn{1}{c}{ Marginals fit } & $\chi^{2}$ & $d f$ & $p^{b}$ \\
\hline 1 & MIAE, NIAE, NMI, NMA, NME & 21.31 & 20 & .379 \\
2 & MIAE, NIAE, NMI, NMA & 21.66 & 22 & .480 \\
3 & MIAE, NIAE, NMI, NME & 22.21 & 22 & .448 \\
4 & MIAE, NIAE, NMA, NME & 22.16 & 22 & .451 \\
5 & MIAE, NIAE, NMI & 22.81 & 24 & $.5<\mathrm{p}<.7$ \\
6 & MIAE, NIAE, NMA & 22.97 & 24 & $.5<\mathrm{p}<.7$ \\
7 & MIAE, NIAE, NME & 23.53 & 24 & .489 \\
8 & MIAE, NIAE, NM & 25.01 & 26 & $.5<\mathrm{p}<.7$ \\
9 & MIAE, NMI, NMA, NAE & 34.91 & 38 & $.5<\mathrm{p}<.75$ \\
10 & MIAE, NMI, NMA, NE & 37.47 & 42 & $>.5$ \\
11 & MIAE, NMI, NAE & 36.09 & 40 & $.5<\mathrm{p}<.75$ \\
12 & MIAE, NMI, NA, NE & 38.86 & 44 & $>.5$ \\
13 & MIAE, NM, NI, NA, NE & 41.82 & 46 & $>.5$ \\
14 & MIAE, NMA, NIE & 35.65 & 40 & $.5<\mathrm{p}<.75$ \\
15 & MIAE, NMA, NI, NE & 39.31 & 44 & $>.5$ \\
16 & MIAE, NMI, NA & 130.29 & 46 & .000 \\
17 & MIAE, NMI, NE & 44.32 & 46 & $>.5$ \\
18 & MIAE, NMA, NI & 131.32 & 46 & .000 \\
19 & MIAE, NMA, NE & 46.35 & 46 & .458 \\
\hline
\end{tabular}

a A total of 2444 cases had nonmissing data on the five variables, out of a possible 3173. The two largest sources of missing data were income $(561$ cases) and the 116 cases who broke off interviews before completing all questions. The table contains $2 \times 2 \times 3 \times 3 \times 3=$ 108 cells all of which contained elements. Parameters measuring effects of polytymous variables are differences between the average log frequency over all levels (the so-called "standard effects" in Goodman, 1971.)

- These probability values are based on the assumption of simple random sampling and have not been adjusted for the clustering of some sample elements into primary areas.

sures in our analysis of the "respondent reaction" variables and method effects. Age and income both have influence on observed method effects; we choose to include age because of a lower missing data rate.

Table 7 presents the results of $\log$ linear analysis on the five-way table including number of problems mentioned $(\mathrm{N})$, mode of administration (M), respondent's age (A), interviewer judgment of the respondent's ability to articulate his thoughts ( $\mathrm{T})$, and of the respondent's apparent interest in the interview (R). ${ }^{9}$ As before, we are interested in the significance of terms that indicate different method effects across categories

${ }^{9}$ The respondent's ability to articulate his thoughts is employed as a three-category variable collapsed from a four-category scale: I-first category on scale-Very Articulate; $2=$ second category on scale: $3=$ third and fourth categories on scale-Limited Vocabulary. Interest is also a three-category collapsing of a four-category scale $(1=$ Very High Interest, $2=$ Above Average Interest, $3=$ Average to Very Low Interest). 
TABLE 7

Log Linear Models Fit to the Five-Way Contingency Table Including Number of Problems Mentioned (N), Mode of Administration (M), Respondent's Age (A), Interviewer Judgment of Respondent's Ability to Articulate Thoughts ( $T$ ), and of Respondent's Apparent Interest in the Interview $(\mathbf{R})^{a}$

\begin{tabular}{llccc}
\hline Model & \multicolumn{1}{c}{ Marginals fit } & $\chi^{2}$ & $d f$ & $p^{b}$ \\
\hline 1 & MATR, NATR, NMA, NMT, NMR & 15.30 & 20 & $.7<\mathrm{p}<.8$ \\
2 & MATR, NATR, NMA, NMT & 19.78 & 22 & $.5<\mathrm{p}<.7$ \\
3 & MATR, NATR, NMA, NMR & 17.63 & 22 & $.7<\mathrm{p}<.8$ \\
4 & MATR, NATR, NMT, NMR & 22.80 & 22 & .41 \\
5 & MATR, NATR, NMA & 20.59 & 24 & $.5<\mathrm{p}<.7$ \\
6 & MATR, NATR, NM & 27.55 & 26 & .381 \\
7 & MATR, NMA, NTR & 38.19 & 40 & $.5<\mathrm{p}<.75$ \\
8 & MATR, NMA, NT, NR & 41.13 & 44 & $>.5$ \\
9 & MATR, NM, NA, NT, NR & 48.52 & 46 & .372 \\
10 & MATR, NMA, NT & 64.59 & 46 & .036 \\
11 & MATR, NMA, NR & 103.94 & 46 & .0002 \\
12 & MATR, NMA & 188.89 & 48 & .000 \\
\hline
\end{tabular}

a A total of 2827 cases had nomissing data on the five variables out of a possible 3173 . The largest sources of missing data were the 116 cases who broke off interviews before completing all questions. The table contains $2 \times 2 \times 3 \times 3 \times 3=108$ cells of which five contained no elements; .5 cases were added to all cells of the table. Parameters measuring effect of polytymous variables are differences between the average log frequency for each level and the average log frequency over all levels (the so-called "standard effects" in Goodman, 1971).

${ }^{b}$ These probability values are based on the assumption of simple random sampling and have not been adjusted for the clustering of some sample elements into primary areas.

of ability to articulate thoughts (T) and interest (R). These terms are NMT and NMR. We want to see if these terms are significant even in the presence of the demographic interaction NMA. Table 7 shows that they are not; model 8 that contains the age interaction NT fits the data better $\left(\chi^{2}=41.13, d f=44\right)$ than alternative models including either or both NMR and NMT. A comparison of the fit of models 8 and 9 tests the significance of age as a predictor of method effects. In the presence of the "respondent reaction" variables, age has significant influence on method effects $\left(x^{2}=7.39, d f=2,0.25<p<.01\right)$. We have already demonstrated in work of Table 6 that other demographic variables can explain these effects. That is, these particular variables obtained from interviewer judgments do not identify any distinct subgroups susceptible to the method effect that are not also identified by age. ${ }^{10}$

${ }^{10}$ It is useful to note that a similar analysis replacing age by income yields the same conclusion. 


\section{SUMMARY}

This analysis began with the observation that there appeared to be a variation in pace between telephone and personal interviews. After verifying that difference empirically, we attempted to track down possible causes and effects of the difference that might relate to the various properties of data collected on the telephone. We first learned that phone interviews tended to yield fewer second mentions on open-ended items. Our analysis than demonstrated that faster interviews tended to collect fewer responses to open-ended items and that the effect of speed was even more pronounced in telephone interviews. Further, there are larger discrepancies between the types of problems mentioned on the phone versus face-to-face among the group that gives fewer mentions.

Given this evidence, we attempted to identify groups which appeared most susceptible to the tendency to give fewer mentions on the telephone. Both demographic variables and measures of the respondent's reaction to the interview were examined. Sex and education were found to be insignificant in the presence of age and income. Higher income groups and younger respondents exhibit the greatest tendency to fewer mentions on the telephone. In the presence of these variables, respondent's selfperceived uneasiness about answering the questions, interviewer's judgments about the respondent's ability to articulate thoughts, and judgments about his interest in the interview all have insignificant effects on the tendency to fewer mentions on the telephone. Age and income appear to be equally useful as discriminators of groups for which the decision regarding mode of administration may be important to the quality of data obtained.

\section{REFERENCES}

Colombotos, J. (1965), "The effects of personal vs. telephone interviews on socially acceptable responses," Public Opinion Quarterly 29, 457-458.

Goodman, L. A. (1971), "The multivariate analysis of qualitative data: Interactions among multiple classifications," Journal of the American Statistical Association 65, 226-256.

Groves, R. M., and R. L. Kahn (forthcoming), Comparing Telephone and Personal Interview Surveys, Academic Press, New York.

Hochstim, J. R. (1967), "A critical comparison of three strategies of collecting data from households," Journal of the American Statistical Association 62, 976-989.

Rogers, T. F. (1976), "Interviews by telephone and in person: Quality of responses and field performance," Public Opinion Quarterly 40, 51-65. 